\title{
RIEGO-ASESOR: GESTIÓN DEL RIESGO HÍDRICO Y PLANIFICACIÓN SOSTENIBLE DEL AGUA EN LA AGRICULTURA
}

\author{
Jaime RIBALAYGUA ${ }^{1 *}$, José Manuel MIRÁS ${ }^{2}$, Rafael Ángel FERRER ${ }^{3}$, Robert \\ MONJO$^{1}$, Diego INTRIGLIOLO ${ }^{2}$, Manuel MARTÍN ${ }^{3}$, Javier PÓRTOLES ${ }^{1}$, Juan \\ José ALARCÓN ${ }^{2}$, Alfredo José AYALA ${ }^{3}$ \\ ${ }^{1}$ Fundación para la Investigación del Clima (FIC). \\ ${ }^{2}$ Centro de Edafología y Biología Aplicada del Segura (CEBAS-CSIC). \\ ${ }^{3}$ Grupo Hispatec Informática Empresarial S.A. \\ *fic@,ficlima.org
}

\section{RESUMEN}

La eficiencia en el uso de los recursos naturales es una prioridad para garantizar la sostenibilidad económica y ambiental de la agricultura. El proyecto Riego-Asesor analiza las relaciones entre la variabilidad climática y las necesidades requeridas en la gestión hídrica agrícola para así diseñar una batería de buenas prácticas de riego en base a pronósticos meteorológicos. El proyecto además desarrolla modelos semimecanicisticos de cultivos que permiten predecir el balance hídrico y simular así el déficit hídrico existente además de cuantificar la respuesta productiva frente al agua aplicada. Un aspecto importante de Riego-Asesor es la implementación de un sistema de predicción meteorológica que se adapta a las características microclimáticas de cada punto. Mediante la implantación de este sistema se esperan importantes beneficios en el ahorro y eficiencia de agua, incrementos de rendimiento y reducción del gasto energético, entre otros, gracias a la mejora de la predicción de la precipitación y de la evapotranspiración de referencia.

Palabras clave: eficiencia, sostenibilidad, agua, riego, agricultura.

\begin{abstract}
The efficient use of natural resources is a priority to ensure economic and environmental sustainability of agriculture. Riego-Asesor project analyzes the relationship between climate variability and the needs required in agricultural water management in order to design a set of good irrigation practices based on weather forecasts. The project also develops semi-mechanistic crop models for predicting water balance and thus simulate the existing water deficit. In addition, these models try to quantifying the productive response to applied water. An important aspect of Riego-Asesor is the implementation of a weather forecasting system that adapts to the microclimatic characteristics of each point. By implementing this system important benefits are expected in water savings and efficiency, yield increases and reduced energy expenditure, among others, through the improved prediction of precipitation and reference evapo-transpiration.
\end{abstract}

Key words: efficiency, sustainability, water, irrigation, agriculture. 


\section{INTRODUCCIÓN}

La agricultura contribuye positivamente al balance comercial de la economía española con un superávit de 8.017 M€ (Magrama, 2014). Esto se debe fundamentalmente a la producción de frutas y hortalizas que se localiza sobre todo en la costa mediterránea. En estas zonas de cultivo, el principal reto al que se enfrenta la agricultura es el de mantener la calidad y seguridad de la producción haciendo frente a una cada vez menor disponibilidad de agua, tanto en cantidad como en calidad, para cubrir las necesidades de los cultivos. Además, debido al cambio climático y a la competencia con otros sectores económicos, es esperable que en los próximos años los recursos hídricos disponibles para la agricultura puedan ser más escasos y caros (IPCC 2014).

Para hacer frente a esta situación, además de continuar con las modernizaciones de los regadíos, es cada vez más importante incrementar la eficiencia en el uso del agua (Estrategia Nacional para la Modernización Sostenible de los Regadíos, Horizonte 2015). En particular, a nivel de parcela, en la actualidad el riego suele aplicarse de forma muy empírica dado que no existen herramientas sofisticadas y de fácil empleo que permitan tomar decisiones sobre la programación del riego. Las investigaciones realizadas en los últimos diez años han puesto a punto nuevas técnicas de riego para el manejo eficiente del agua en parcela (Ballester et al., 2014; Pedrero et al., 2014), que sin embargo no se aplican a nivel comercial debido a: 1) carencia de capacitación, 2) difícil acceso a la información y 3) la complejidad que conlleva materializar las estrategias de riego eficiente en fincas comerciales.

Ante este reto científico-técnico, el proyecto Riego-Asesor tiene como objetivo desarrollar y validar en condiciones de campo un sistema integral de asesoramiento virtual (on-line) que ponga a disposición de los usuarios finales un sistema de toma de decisiones sobre el riego, centrado en cultivos hortofrutícolas típicos de la agricultura intensiva del litoral mediterráneo. Este proyecto permitirá obtener nuevos conocimientos sobre técnicas de riego eficiente y agrometeorología.

Desde un punto de visto meteorológico, este reto requiere combinar técnicas de modelización numérica y aproximaciones estadísticas, que habitualmente se apoyan de registros climáticos. Además de las observaciones históricas, si además se utilizan las observaciones en tiempo cuasi-real, los resultados mejoran notablemente, mediante la aplicación de técnicas de "error feedback-correction" (Zhuet al., 2007; Si et al., 2015).

El presente estudio tiene como objetivo difundir los principales resultados de la primera fase del proyecto, que se corresponde con el desarrollo e implementación operativa de un Sistema de Predicción Meteorológica basado en correcciones a tiempo cuasi-real y funciones de transferencia (Monjo et al., 2014).

\section{2. ÁREA DE ESTUDIO Y DATOS}

\section{1. Área piloto y observaciones}

Para esta primera fase de la investigación de Riego-Asesor, se tomó un área piloto correspondiente a la parcela con rotaciones hortícolas del campo de Cartagena situada en las coordenadas $37^{\circ} 45^{\prime} 26.4^{\prime \prime} \mathrm{N}, 0^{\circ} 52^{\prime} 26.7^{\prime \prime} \mathrm{W}$. Como fuente de observaciones meteorológicas de apoyo, se consideraron 5 observatorios (Tabla 1) pertenecientes a la red del Sistema de Información Agraria de Murcia (SIAM-IMIDA, http://siam.imida.es/). 
En particular, se tomaron un total de 12 variables meteorológicas (Tabla 2), con resolución diaria y horaria. Además, se tomaron datos de precipitación observada de la red de 703 pluviómetros automáticos de la Agencia Estatal de Meteorología (AEMET), con una resolución horaria. En esta primera fase, el período analizado se corresponde con abril y mayo de 2016 .

\begin{tabular}{ccccc}
\hline ID & Lon $\left(^{\circ}\right)$ & Lat $\left(^{\circ}\right)$ & Nombre & Población \\
\hline TP22 & $-0,819578$ & 37,791694 & Santiago de la Rivera & San Javier \\
TP52 & $-0,883542$ & 37,848119 & El Mirador & San Javier \\
TP42 & $-0,898506$ & 37,773858 & Torre Blanca & Torre Pacheco \\
TP73 & $-0,931481$ & 37,823894 & Los Infiernos & Torre Pacheco \\
TP91 & $-0,986672$ & 37,747725 & Torre Pacheco & Torre Pacheco \\
\hline
\end{tabular}

Tabla 1: Estaciones meteorológicas empleadas para el área piloto. Las coordenadas se muestran tanto en grados como en metros UTM-ETRS89.

\begin{tabular}{ccc}
\hline Variable & Longitud de la serie (años) & Influencia sobre la ETo \\
\hline Temperatura máxima & 3 & Media \\
Temperatura mínima & 3 & Media \\
Temperatura media & 3 & Baja \\
Humedad relativa máxima & 3 & Media \\
Humedad relativa mínima & 3 & Media \\
Humedad relativa media & 3 & Baja \\
Velocidad del viento media & 3 & Alta \\
Velocidad del viento máxima & 3 & Baja \\
Precipitación & 3 & Media \\
Radiación solar & 3 & Alta \\
Presión atmosférica & 1 & Baja \\
Temperatura de rocío & 1 & Baja \\
\hline
\end{tabular}

Tabla 2: Relación de las variables meteorológicas consideradas para el estudio.

\subsection{Simulaciones de partida}

Se emplearon tres simulaciones numéricas con salida $00 \mathrm{Z}$ diaria correspondientes al HIRLAM 0.05, el HIRLAM 0.16 y el ECMWF 0.25. Los horizontes de predicción que se emplean son $36 \mathrm{~h}, 72 \mathrm{~h}$ y $240 \mathrm{~h}$, respectivamente. La simulación de las variables meteorológicas (Tabla 2) se genera a escala local (parcela) con una resolución horaria para el horizonte de 3 días y con una resolución 6-horaria hasta 10 días.

Para el horizonte inferior a 6 horas, se emplean, además, datos de reflectividad de los radares meteorológicos de la Agencia Estatal de Meteorología (AEMET), con una 
resolución espacial de $1 \mathrm{~km}$ y temporal de 10 minutos. Especialmente se emplean los productos CAPPI del radar de Murcia y Almería.

\section{METODOLOGÍA}

\subsection{Predicción meteorológica de temperatura y humedad}

Una vez identificadas las variables meteorológicas de interés para el proyecto, se desarrolló y validó un Sistema de Predicción Meteorológica (SPM). Para ello se combinaron técnicas de predicción numérica y técnicas de "error feedback-correction" o corrección móvil, así como funciones de transferencia estadística (Monjo et al., 2014).

Para el caso de las temperaturas (mínima, máxima, media y temperatura de rocío), se realizan transferencias lineales de la distribución de probabilidad observada durante los 7 días previos. Por ejemplo, si consideramos una distribución gaussiana de la temperatura, la temperatura simulada final será:

$$
T_{\text {sim }}(D)=\bar{T}_{\text {obs }}(-1,-N)+\frac{\sigma_{\text {obs }}(-1,-N)}{\sigma_{\text {pred }}(-1,-N)}\left[T_{\text {pred }}(D)-\bar{T}_{\text {pred }}(-1,-N)\right]
$$

Ec. 1

donde $T, \bar{T}$ y $\sigma$ son respectivamente la temperatura en un día, la temperatura media y la desviación típica: Observada (obs), predicha por los modelos numéricos (pred) y simulada con el método utilizado $(\mathrm{sim})$. Por otro lado, la información de los paréntesis $D,-1$, y $-N$ representan el horizonte de $D$ días y los valores de hace 1 hasta $\mathrm{N}$ días, respectivamente.

La humedad relativa máxima y mínima se obtiene mediante la relación de Clausius-Clapeyron a partir de la temperatura $(T)$ y la temperatura de rocío $\left(T_{d}\right)$ :

$$
H R=100 \exp \left[5419.11\left(\frac{1}{T}-\frac{1}{T_{d}}\right)\right]
$$

La velocidad media y máxima del viento se corrige en base a las distribuciones de Weibull y Rayleigh, mientras que la precipitación se redistribuye según los modelos de probabilidad de Monjo et al. (2014, 2016).

El método de corrección por transferencia estadística fue verificado tanto para la temperatura como la humedad relativa, de acuerdo con una comparación del error absoluto medio que se obtiene antes y después de aplicar el método. De ese modo, se pudo obtener un indicador de habilidad o de mejora.

\subsection{Predicción meteorológica de la lluvia}

La predicción de la precipitación a menos de 6 horas se corrige en base a una proyección de la evolución (desplazamiento e intensificación/disipación) de los sistemas de precipitación, especialmente frontales. Para el horizonte de 1 a 2 horas, se incorpora además una corrección basada en el movimiento de los núcleos convectivos, identificados mediante $k$-means clustering (MacKay, 2003). Los datos de reflectividad del radar $(Z)$ se traducen en una estimación de intensidad media $(R)$ de acuerdo con la relación ZR de Marshall-Palmer (1948): 


$$
Z=a R^{b}
$$

donde $a$ y $b$ son parámetros de ajuste.

Esta relación se ajusta con los datos observados de precipitación de las horas previas y se incorpora como una tercera dimensión en una interpolación espacial de las observaciones, del tipo Thin-Plate-Spline (TPS). Para un mejor ajuste de la relación reflectividad-intensidad, se efectúa una interpolación temporal de la reflectividad hasta resolución 1-minutal. La bondad del método fue evaluada mediante una verificación cruzada de la inteporlación espacial, y se obtuvo un error medio absoluto. Todos los cálculos se han realizado mediante lenguaje de programación R (Muenchen and Hilbe, 2010).

Para este primer trabajo, la presión atmosférica no se corrige ya que los modelos de simulación de la evapotranspiración de referencia son poco sensibles a esta variable.

\section{RESULTADOS Y DISCUSIÓN}

\subsection{Predicción de temperatura}

La predicción de la temperatura realizada a partir del HIRLAM presenta errores absolutos medios que en general son bajos ya que oscilan entre 1 y $2^{\circ} \mathrm{C}$ para el área de estudio. Sin embargo, en algunos casos esos errores medios alcanzan $\operatorname{los} 3^{\circ} \mathrm{C}$, especialmente en temperaturas máximas (Fig. 1). Después de aplicar el método de corrección móvil o feedback-correction, los errores medios se reducen en promedio hasta $1^{\circ} \mathrm{C}$. Los casos con errores extremos también mejoran notablemente: Si en algunos días se producían como máximo errores de $6^{\circ} \mathrm{C}$, con el método aplicado esos errores se reducen hasta 2 o $3^{\circ} \mathrm{C}$ (Fig. 1 y 2 ).
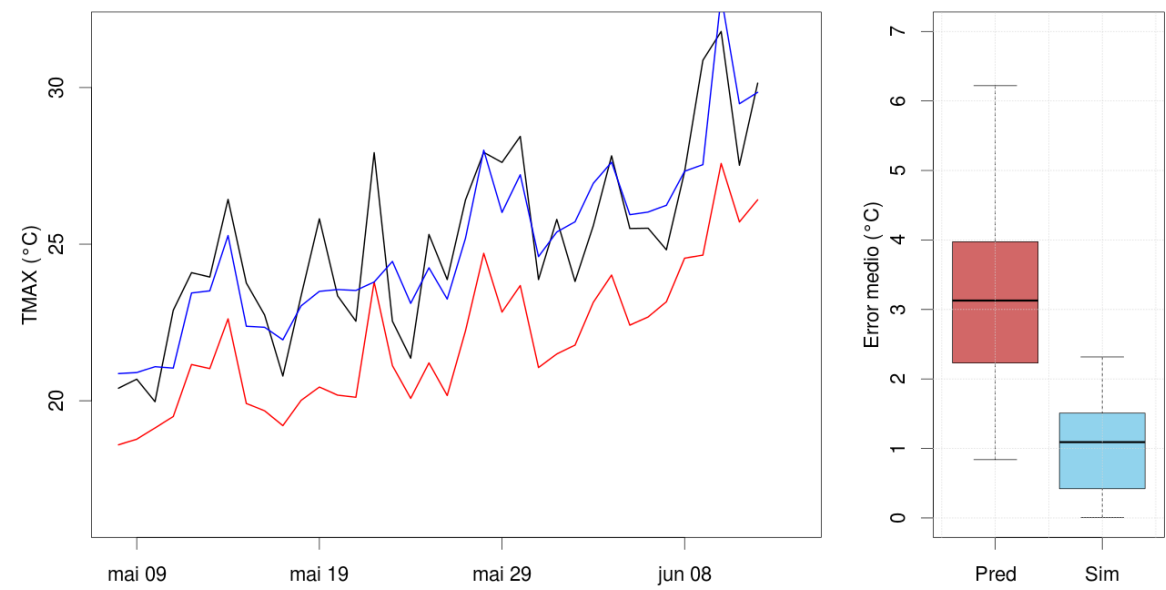

Fig. 1. (Izda) Temperatura máxima observada (línea negra), predicha (línea roja) y simulada con el método propuesto (línea azul), para Santiago de la Rivera (TP22). (Dcha) Diagrama de cajas del error absoluto medio obtenido según la predicción (caja roja) y la simulada (caja azul). 

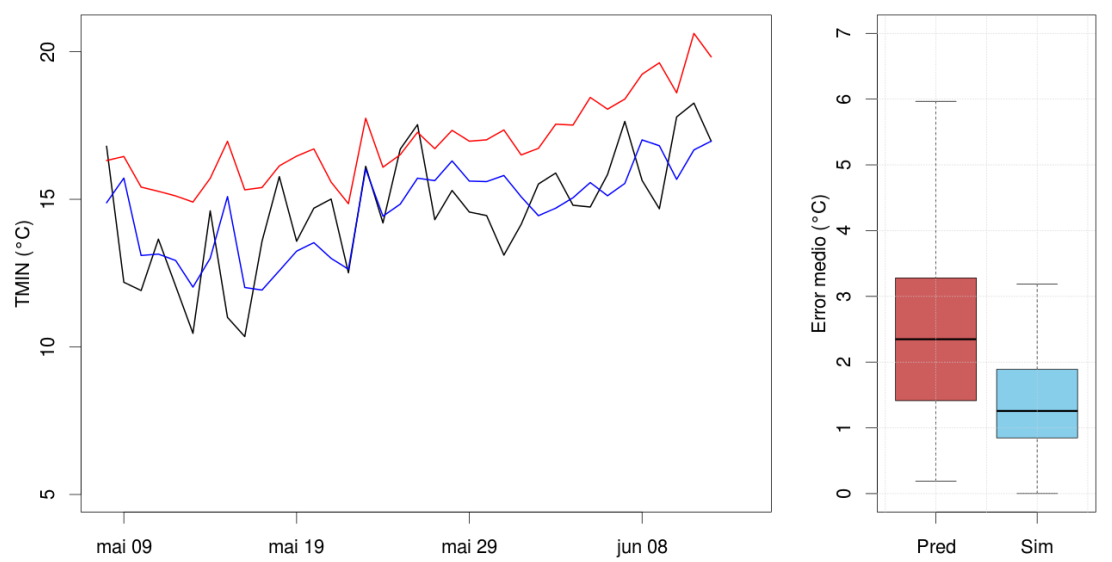

Fig. 2. (Izda) Temperatura minima observada (linea negra), predicha (linea roja) y simulada con el método propuesto (línea azul), para Santiago de la Rivera (TP22). (Dcha) Diagrama de cajas del error absoluto medio obtenido según la predicción (caja roja) y la simulada (caja azul).

\subsection{Predicción de humedad}

En cuanto a la humedad relativa, los mayores errores de la predicción inicial se encuentran en la simulación de la humedad mínima, con desviaciones que en algunos casos son superiores al 15\%. En el caso de la humedad máxima, los errores medios son en todos los casos inferiores al 10\%. Después de aplicar la corrección móvil, el error medio se reduce por debajo del $5 \%$ en humedades máximas y por debajo del $10 \%$ en humedades mínimas (Fig. 3 y 4 ).
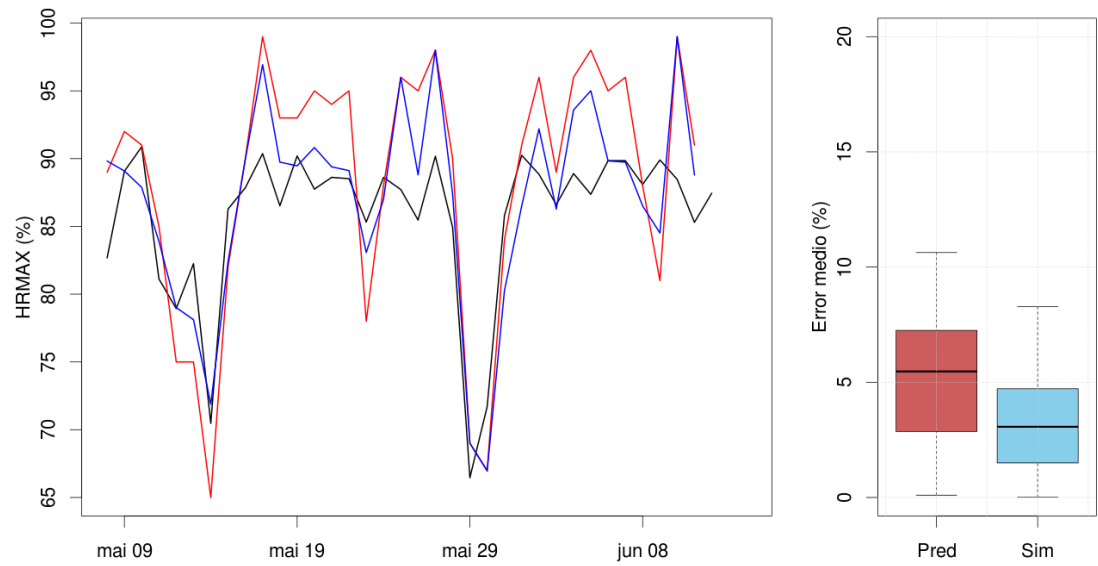

Figura 3. (Izda) Humedad máxima observada (linea negra), predicha (linea roja) y simulada con el método propuesto (línea azul), para Santiago de la Rivera (TP22). (Dcha) Diagrama de cajas del error absoluto medio obtenido según la predicción (caja roja) y la simulada (caja azul). 

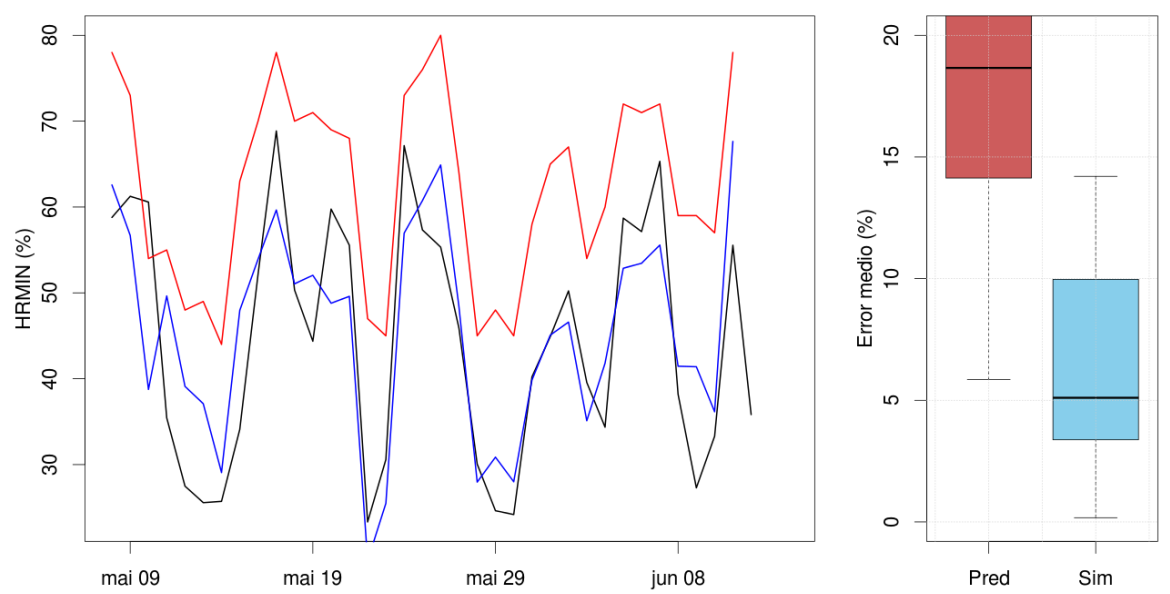

Fig. 4. (Izda) Humedad mínima observada (línea negra), predicha (línea roja) y simulada con el método propuesto (linea azul), para Santiago de la Rivera (TP22). (Dcha) Diagrama de cajas del error absoluto medio obtenido según la predicción (caja roja) y la simulada (caja azul).

\subsection{Predicción de precipitación}

La combinación de las observaciones locales a tiempo cuasi-real y la reflectividad obtenida por los radares meteorológicos de la AEMET proporciona una buena estimación de la distribución espacial de la lluvia. Según la verificación cruzada, los errores de la interpolación de las observaciones se reducen del 20\% al 14\% al incorporar los datos de los radares (Fig. 5).

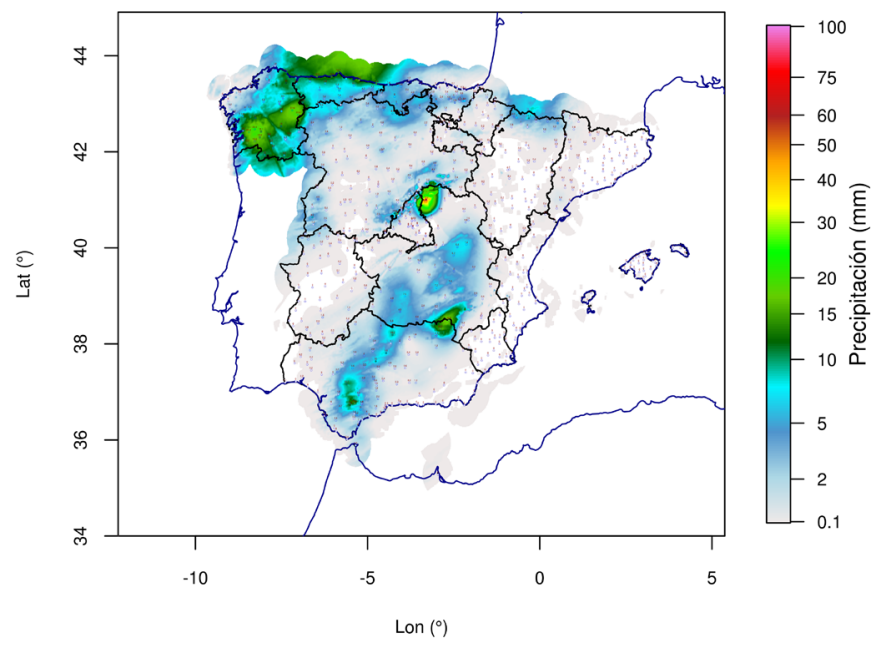

Fig. 5. Estimación de la lluvia para el 14 de abril de 2016 obtenida a partir de la combinación de los radares meteorológicos y la red de pluviómetros automáticos de la AEMET. 
Los errores relativos son ligeramente superiores para situaciones de tormentas debido a la gran variabilidad espacial que estas provocan. Además, existe un importante error sistemático $(18 \%)$ en la estimación del área afectada por precipitaciones significativas $(>0.1 \mathrm{~mm})$, aunque el error disminuye cuando consideramos un umbral de $1 \mathrm{~mm}$ (error del 12\%).

\section{CONCLUSIONES}

Las predicciones de partida de la temperatura y la humedad son en general adecuadas para su uso en predicción operativa de riesgos tales como las olas de calor y periodos cortos de sequía. Sin embargo, para el uso agrícola pueden ser insuficientes debido a las necesidades de gran precisión en la estimación del balance hídrico.

Combinando técnicas de predicción numérica y técnicas de "error feedback-correction", se logra reducir notablemente el error sistemático de la temperatura y la humedad relativa. Para el caso de la temperatura, los errores medios finales son cercanos a $1^{\circ} \mathrm{C}$ en la mayoría de los casos. Para la humedad relativa, los errores son ligeramente superiores al 5\% en humedades mínimas y algo inferior para las máximas.

Del mismo modo, combinando observaciones en tiempo cuasi-real de pluviómetros y reflectividad del radar, se obtuvo una estimación adecuada de la distribución espacial de la lluvia. De hecho, el error medio de la interpolación se redujo del 20 al 14\%.

Mediante la implantación de este sistema se esperan importantes beneficios en el ahorro y eficiencia de agua, incrementos de rendimiento y reducción del gasto energético, entre otros.

\section{AGRADECIMIENTOS}

El proyecto Riego-Asesor está financiado por el MINECO con co-financiación FEDER a través del proyecto Retos Colaboración RTC-2015-3453-2: "Asesor virtual para la ayuda a la toma de decisiones sobre estrategias de riego sostenibles".

\section{REFERENCIAS}

Ballester, C.; Castel, J.; Abd El-Mageed, T.A.; Castel, J.R.; Intrigliolo, D.S. (2014). Long-term response of 'Clementina de Nules' citrus trees to summer regulated deficit irrigation. Agric Water Manag, 138, 78-84.

IPCC (2014). Fifth Assessment Report. Climate Change 2014: Impacts, Adaptation, and Vulnerability. Part B: Regional Aspects. Europe.

Pedrero, F.; Maestre-Valero, J.F.; Mounzer, O.; Alarcón, J.J.; Nicolás E. (2014): Physiological and agronomic mandarin trees performance under saline reclaimed water combined with regulated deficit irrigation. Agric Water Manag, 146, 228-237.

MAGRAMA (2014). Análisis del comercio exterior agrario y pesquero. Ministerio de Agricultura, Alimentación y Medio Ambiente (MAGRAMA). Annual Report 2013. Enlace: http:/www.magrama.gob.es/es/ministerio/servicios/analisis-yprospectiva/Annual_reports.aspx

MacKay, D. (2003). An Example Inference Task: Clustering. Information Theory, Inference and Learning Algorithms. Cambridge University Press. 284-292. ISBN 0-521-64298-1. MR 2012999. 
Marshall, J. S., and W. McK. Palmer 1948.The distribution of raindrops with size.J. Meteor.5. 165-166.

Monjo, R.; Caselles, V.; Chust, G. (2014). Probabilistic correction of RCM precipitation in the Basque Country (Northern Spain). Theor Appl Climatol, 117, 317-329. doi: 10.1007/s00704-013-1008-8.

Monjo, R.; Gaitán, E.; Pórtoles, J.; Ribalaygua, J.; Torres, L. (2016): Changes in extreme precipitation over Spain using statistical downscaling of CMIP5 projections. Int J Climatol, 36, 757-769. doi: 10.1002/joc.4380.

Muenchen, R.A.; Hilbe, J.M. (2010). R for Stata Users. Statistics and Computing. Springer, ISBN: 978-1-4419-1317-3.

Si, W, Bao W, Gupta HV (2015). Updating real-time flood forecasts via the dynamic system response curve method. Water Resour Res, 51, 5128-5144. doi:10.1002/2015WR017234.

Zhu, D; Cheng, D; Robert P. Broadwater, RP; Scirbona, C (2007). Storm modeling for prediction of power distribution system outages. Electric Power Systems Research, 77, 973-979. 